\title{
REABILITAÇÃO FISIOTERAPÊUTICA DE PACIENTES COM SEQUELAS DE ACIDENTE VASCULAR CEREBRAL: UMA REVISÃO DE LITERATURA
}

\section{PHYSIOTHERAPEUTIC REHABILITATION OF PATIENTS WITH SEQUELS OF CEREBRAL ACCIDENT: A LITERATURE REVIEW}

\author{
Antônio Levi Sampaio de Araújo ${ }^{1}$ \\ Francisco Weverton Carneiro Gomes ${ }^{2}$ \\ Gabriel Pereira Fidelis ${ }^{3}$ \\ Jefferson Washington de Souza Rodrigues ${ }^{4}$ \\ José Valdilânio Virgulino Procópio ${ }^{5}$ \\ Marcus Vinícius Leite Batista Lacerda ${ }^{6}$
}

RESUMO: Introdução: O Acidente Vascular Cerebral (AVC) constitui-se em uma sequência de eventos neurológicos agudos, causado pelo bloqueio vascular que dá origem a isquemia em determinada área encefálica ou pela ruptura em decorrência do enfraquecimento dos vasos sanguíneos, ocorrendo uma hemorragia cerebral. Objetivos: Identificar as principais técnicas fisioterapêuticas utilizadas na reabilitação de pacientes com sequelas de AVC, além de apresentar os resultados das técnicas mais eficazes na reabilitação destes pacientes. Materiais e métodos: Utilizou-se de uma revisão integrativa da literatura com abordagem descritiva. A coleta dos artigos necessitou-se da utilização dos seguintes bancos de dados, como: Medical Literature Analysis and Retrieval System Online (MedLine), Scientific Electronic Library (SciELO), Literatura Latino-Americana em Ciências da Saúde (LILACS), Physiotherapy Evidence Data Base (PEDro) e Biblioteca Virtual em Saúde (BVS). Resultados: Após a captação dos artigos foi encontrado as principais

\footnotetext{
${ }^{1}$ Acadêmico do $6^{\circ}$ período do curso de Medicina, Faculdade Santa Maria, Cajazeiras - Paraiba, Brasil. E- mail: lev_sampaioaa@outlook.com.

${ }_{2}$ Acadêmico do $\overline{6}^{\circ}$ período do curso de Medicina, Faculdade Santa Maria, Cajazeiras - Paraiba, Brasil. E- mail: dr.weverton1876@ gmail.com.

${ }^{3}$ Acadêmico do $6^{\circ}$ período do curso de Medicina, Faculdade Santa Maria, Cajazeiras - Paraiba, Brasil. E- mail: gabrielfideliss@gmail.com.

${ }^{4}$ Acadêmico do $6^{\circ}$ período do curso de Medicina, Faculdade Santa Maria, Cajazeiras - Paraiba, Brasil. E- mail: jefferson.4545@hotmail.com.

${ }^{5}$ Acadêmico do $6^{\circ}$ período do curso de Medicina, Faculdade Santa Maria, Cajazeiras - PB, Brasil. Email: valdilaniofsm@gmail.com.

${ }^{6}$ Acadêmico do $5^{\circ}$ período do curso de Medicina, Faculdade Santa Maria, Cajazeiras - Paraiba, Brasil. E- mail: vinicius.b.lacerda@gmail.com.
} 
técnicas fisioterapêuticas utilizadas na reabilitação de pacientes com sequelas de AVC foram o kinesio taping, a eletroterapia, a crioterapia, a terapia espelho, o uso da bola suíça e a cinesioterapia, melhorando as limitações dos pacientes e melhorando sua qualidade vida. Conclusão: O Kinesio Taping e a eletroestimulação foram os tratamentos mais utilizadas dentro dos artigos pesquisados trazendo benefícios desde à diminuição da subluxação do ombro até o desempenho funcional da marcha.

Palavras chave: Reabilitação. Acidente Vascular Cerebral. Sequelas.

ABSTRACT: Introduction: Stroke is a sequence of acute neurological events, caused by vascular blockage that causes ischemia in a certain area of the brain or by rupture as a result of weakening of the blood vessels, resulting in cerebral hemorrhage. Objectives: To identify the main physiotherapeutic techniques used in the rehabilitation of patients with stroke sequelae, besides presenting the results of the most effective techniques in the rehabilitation of these patients. Materials and methods: We used an integrative literature review with a descriptive approach. The collection of articles required the use of the following databases, such as: Medical Literature Analysis and Retrieval System Online (MedLine), Scientific Electronic Library (SciELO), Latin American Literature in Health Sciences (LILACS), Physiotherapy Evidence Data Base (PEDro) and Virtual Health Library (VHL). Results: After capturing the articles, it was found that the main physiotherapeutic techniques used in the rehabilitation of patients with stroke sequelae were kinesio taping, electrotherapy, cryotherapy, mirror therapy, Swiss ball use and kinesiotherapy, improving limitations of patients and improving their quality of life. Conclusion: Kinesio Taping and electrostimulation were the most used treatments within the researched articles, bringing benefits from the reduction of shoulder subluxation to gait functional performance.

Keywords: Rehabilitation. Stroke. Sequels. 


\section{INTRODUÇÃO}

O Acidente Vascular Cerebral (AVC) é uma síndrome neurológica que consiste em um rápido desenvolvimento de distúrbios clínicos focais na atividade cerebral, global em relação ao coma, que se mantem mais de 24 horas ou levam à morte sem outra razão aparente que não a de origem vascular. O AVC pode ser do tipo Isquêmico ou Hemorrágico (BOTELHO et al., 2016).

$\mathrm{Na}$ fase inicial é de grande importância a agilidade neste caso pode impossibilitar a necrose do tecido cerebral. Portanto, é indispensável algumas tecnologias possíveis no tempo certo, tal como a efetuação da tomografia computadorizada entre quatro horas e meia depois de o início dos sintomas (ROLIM; MARTINS, 2011).

É imprescindível a importância do diagnóstico ainda no começo do referente Acidente Vascular Cerebral, pois essa é uma patologia neurológica que compromete as principais funções estabelecidas pelos nervos do corpo (funções motoras). Em relação a esse contexto, apresenta- se o posicionamento de Abramczuk; Villela (2009). Trata-se ainda de uma das maiores causas de inabilitação de indivíduos com idade maior que 50 anos, tornando-se responsáveis por $10 \%$ dos resultados de mortalidade, $32,6 \%$ dos óbitos com motivos vasculares e $40 \%$ das aposentadorias antecipadas no Brasil.

No que tange a mortalidade em relação ao AVC é a doença que mais causa mortes e incapacidades no mundo, sendo assim é também um dos principais motivos de internações. Os números de mortalidade por AVC especificamente no Brasil, estão entre uns dos maiores dentre os países da América Latina, as mulheres são as principais atingidas, em relação ao número de óbitos (GARRITANO et al., 2012).

Afinal, quando não leva a óbito, essa patologia pode levar a incapacidade parcial ou completa do paciente, com pertinentes sequelas. Mostra que a prevenção desta patologia é importante, não apenas para assegurar a melhoria de vida aos 
afetados e seus familiares, bem como para preservar custos com a hospitalização, que é mais difícil, por causa da medicina avançada (BOCCHI; ANGELO, 2004).

A fisioterapia possui diversos recursos terapêuticos que possuem efeitos positivos no tratamento do AVC, como: a cinesioterapia, que são exercícios físicos terapêuticos; hidroterapia (exercícios aquáticos); equoterapia (utiliza o auxílio de equinos); massoterapia (técnicas que mobilizam os tecidos superficiais e profundos); termoterapia e eletroterapia (LEVY; OLIVEIRA, 2003).

A dada pesquisa surge do interesse pessoal em poder trazer maiores conhecimentos a respeito do AVC, que é uma patologia que alveja a população brasileira, e sendo este um dos principais problemas que causam mortes e internações no país.

Assim, esse estudo procurou pesquisar sobre esse assunto para que possam ser debatidos melhores controles e tratamentos dos fatores agravantes dessa patologia, no intuito de trazer incentivos para os profissionais, proporcionando acesso a resultados relevantes de pessoas, e produzindo um sabor crítico sobre o assunto.

Tendo em vista a temática abordada, objetiva-se realizar uma revisão de literatura acerca da reabilitação de pacientes com sequelas de AVC, além de quantificar 0 número de evidências cientificas que abordem 0 tratamento fisioterapêutico de pacientes com sequelas de AVC e apresentar os resultados das técnicas utilizadas na reabilitação destes pacientes.

\section{MATERIAIS E MÉTODOS}

\section{Tipo de estudo}

O presente estudo trata-se de uma revisão de literatura integrativa, de caráter descritivo e exploratório. Segundo Mendes (2008) a pesquisa de revisão literatura é um método de busca, investigação, definição de um corpo do entendimento que vai 
em busca de uma determinada resposta de uma pergunta objetiva, cujo objetivo primordial é alcançar um profundo entendimento de um determinado acontecimento, fundamentando-se em pesquisas anteriores.

\section{Período e local estudo}

Esta pesquisa partiu de um levantamento bibliográfico de evidências científicas publicadas nas bases de Medical Literature Analysis and Retrieval System Online (MedLine), Scientific Electronic Library Online (SciELO), Physiotherapy Evidence Data Base (PEDro), Biblioteca Virtual em Saúde (BVS), nos últimos Dez (10) anos (2009 - 2019).

Para facilitar o acesso aos artigos utilizaram-se alguns descritores, como: Reabilitação. AVC. Sequelas, todos disponíveis nos Descritores em Ciência da Saúde (DeCS) e utilizando o descritor booleano AND.

O período que compreendeu a coleta de dados foi entre os meses de fevereiro a abril de 2019.

\section{Critérios de Elegibilidade}

Para a escolha dos artigos acrescentados ao presente estudo, alguns critérios de inclusão foram feitos, sendo eles: artigos publicados entre o ano 2009 a 2019, que estivessem na língua portuguesa e que tinham como participantes dos estudos pessoas com AVC.

Assim, foram excluídos artigos secundários, revisões bibliográficas, sistemáticas, integrativas, artigos pagos, trabalhos que apresentava apenas 0 resumo publicado (incompleto) e aqueles que o tratamento realizado para o AVC não foi feito por fisioterapeutas. 


\section{Análise de Dados}

Os dados foram analisados e divididos de acordo com o ano e a relevância da pesquisa, e posteriormente apresentadas em gráficos confeccionados pelo programa Microsoft Office Excel 2010 para melhor entendimento da pesquisa.

\section{Aspectos legais e éticos}

Devido não terem sido realizados encontros, entrevistas ou investigações com pacientes, familiares e/ou profissionais de saúde, não foi necessária a submissão desta pesquisa científica ao Comitê de Ética em Pesquisa da Instituição conforme a Resolução 510/16 do Conselho Nacional de Saúde.

\section{RESULTADOS E DISCUSSÕES}

Durante o período de pesquisa dos artigos obteve-se 245 artigos, desses após passar pela triagem a partir dos critérios de inclusão estabelecidos foram selecionados 7 . Sendo assim, foram excluídos no total de 238 evidências científicas.

Além disso, fez-se um fluxograma mostrando as etapas e as quantidades ( $n$ ) de artigos encontrados e excluídos para cada banco de dados, conforme mostra a Figura 1. 
Figura 1 - Fluxograma da captação dos artigos selecionados.

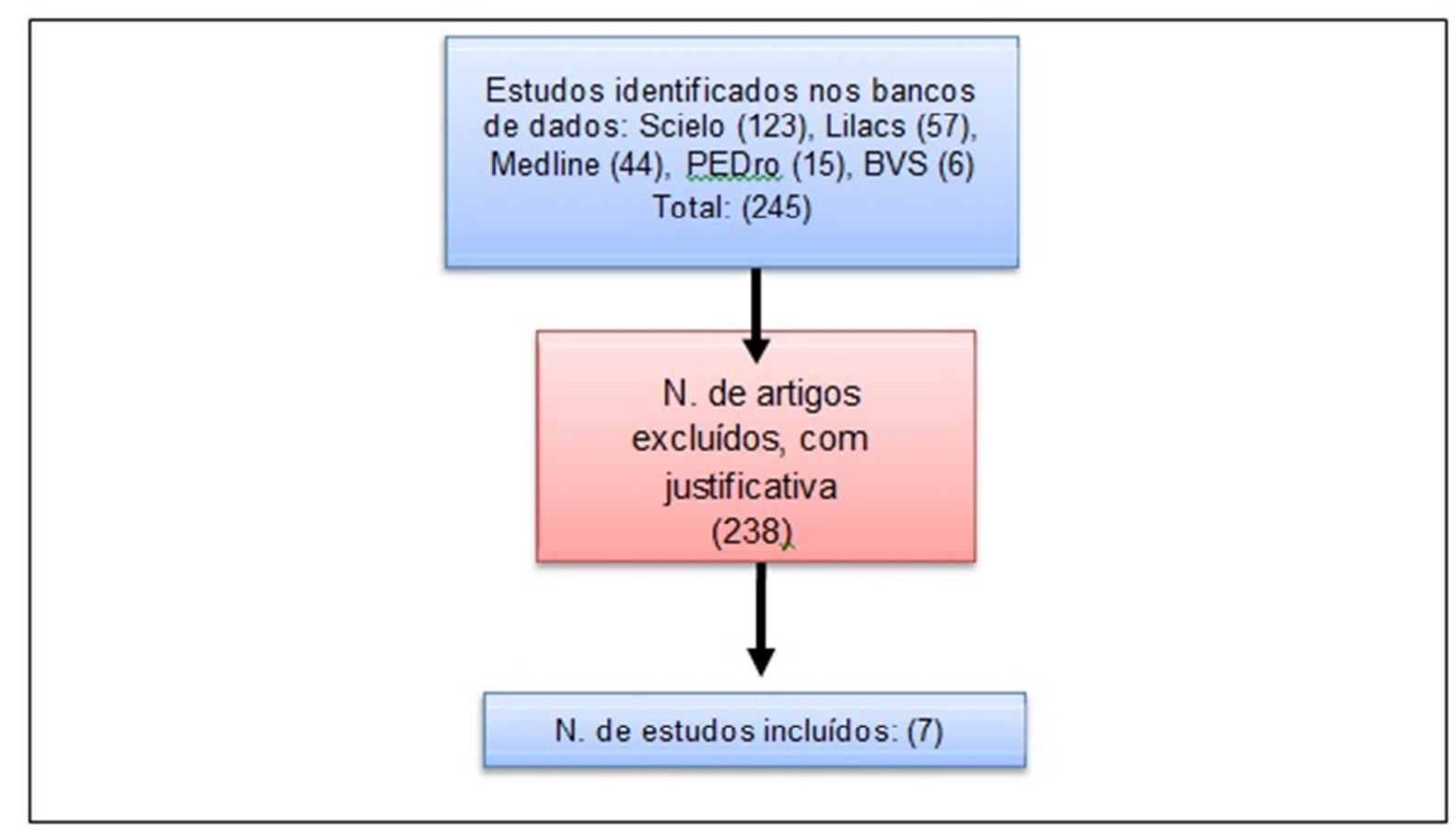

Fonte: Dados da pesquisa (2019).

Apresentando os resultados da presente pesquisa, estão dispostos no quadro 1 , as técnicas fisioterapêuticas utilizadas na reabilitação de pacientes com sequelas de AVC. 
Quadro 1 - Técnicas fisioterapêuticas utilizadas na reabilitação de pacientes com sequelas de AVC.

\begin{tabular}{|c|c|c|c|}
\hline AUTOR/ANO & TÍTULO & OBJETIVOS & CONCLUSÃO \\
\hline & $\begin{array}{l}\text { A influência da } \\
\text { Kinesio Taping } \\
\text { no tratamento } \\
\text { l. } \text { da subluxação } \\
\text { de ombro no } \\
\text { Acidente } \\
\text { Vascular } \\
\text { Cerebral }\end{array}$ & $\begin{array}{l}\text { gerificar a influênc } \\
\text { odo uso da técni } \\
\text { do Kinesio Taping } \\
\text { otratamento } \\
\text { oubluxação } \\
\text { ombro em pacient } \\
\text { com AVC }\end{array}$ & 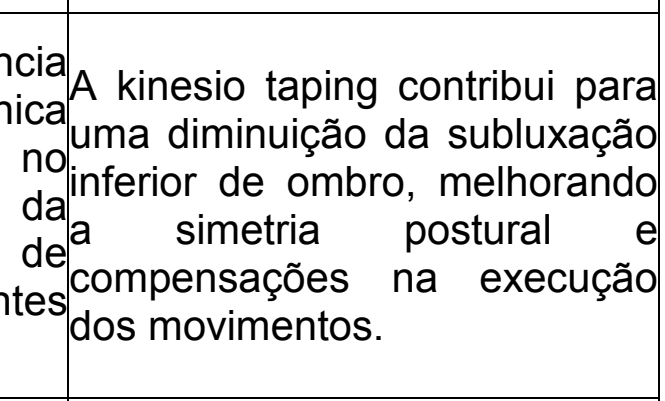 \\
\hline & $\begin{array}{l}\text { Eletroestimulaç } \\
\text { ão } \\
\text { Crioterapia } \\
\text { para } \\
\text { espasticidade } \\
\text { em pacientes } \\
\text { acometidos por } \\
\text { Acidente } \\
\text { Vascular } \\
\text { Cerebral }\end{array}$ & $\begin{array}{l}\text { Avaliar entre } \\
\text { técnicas de EENM } \\
\text { crioterapia, aque } \\
\text { que obtêm } \\
\text { diminuição } \\
\text { espasticidade } \\
\text { muscular e } \\
\text { melhora da ativida } \\
\text { funcional. }\end{array}$ & $\begin{array}{l}\text { as } \\
\mathrm{M} \text { eA crioterapia neuromuscular } \\
\text { uelaquanto a EENM promoveu } \\
\text { aredução da atividade elétrica } \\
\text { damuscular de quadríceps e } \\
\text { melhora no desempenho } \\
\text { afuncional da marcha nos três } \\
\text { adepacientes estudados. }\end{array}$ \\
\hline $\begin{array}{l}\text { Correia } \\
\text { al., (2010) }\end{array}$ & $\begin{array}{l}\text { Crioterapia } \\
\text { cinesioterapia } \\
\text { no memb } \\
\text { superior } \\
\text { espastico } \\
\text { acidente } \\
\text { vascular } \\
\text { cerebral. }\end{array}$ & $\left\{\begin{array}{l}\text { Avaliar o efeito } \\
\text { crioterápia } \\
\text { associada } \\
\text { cinesioterapia } \\
\text { padrão postur } \\
\text { flexor e no grau } \\
\text { espasticidade } \\
\text { membro superior } \\
\text { pacientes com AVC }\end{array}\right.$ & $\begin{array}{l}\text { Crioterapia associada à } \\
\text { cinesioterapia parece ser efi } \\
\text { ciente na diminuição do tônus } \\
\text { damuscular no membro superior } \\
\text { espástico de pacientes com } \\
\text { àdiagnóstico de acidente } \\
\text { novascular cerebral, permitindo } \\
\text { uralredução no grau de } \\
\text { deespasticidade e melhora no } \\
\text { dopadrão postural estático do } \\
\text { demembro superior observada a } \\
\text { /C. partir da redução no grau de fl } \\
\text { exão das articulações } \\
\text { metacarpofalângeas } \\
\text { interfalângeas. }\end{array}$ \\
\hline
\end{tabular}




\begin{tabular}{|c|c|c|}
\hline $\begin{array}{l}\text { Apolinario } \\
\text { al., (2014) }\end{array}$ & et. $\begin{array}{l}\text { Kinesio Taping } \\
\text { como auxiliar } \\
\text { na recuperação } \\
\text { pós- AVC nocientes } \\
\text { membro } \\
\text { superior } \\
\text { parético: Um } \\
\text { ensaio clinico. }\end{array}$ & 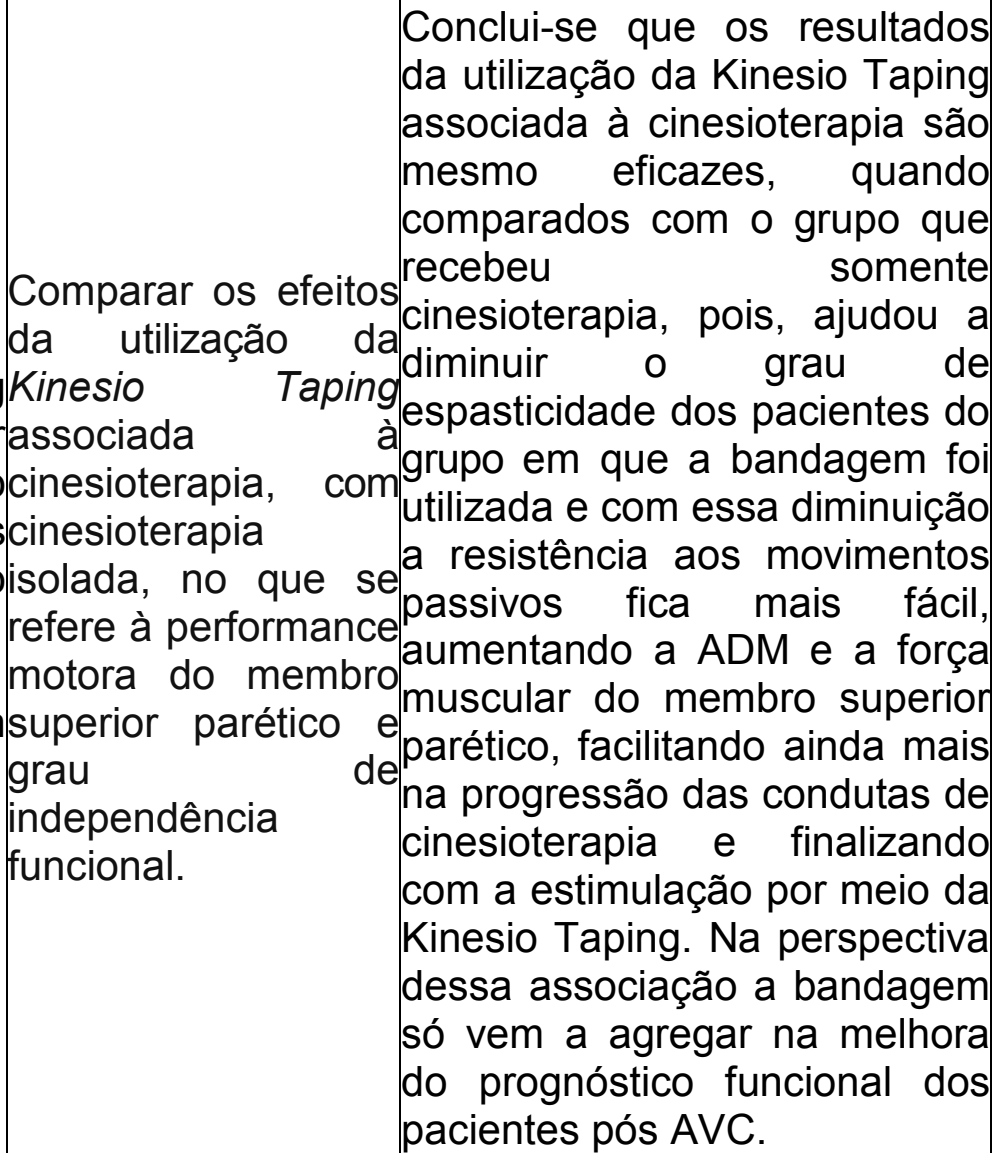 \\
\hline $\begin{array}{l}\text { Santos } \\
\text { Ferreira } \\
(2015)\end{array}$ & $\begin{array}{l}\text { Cinesioterapia } \\
\text { neuro funcional } \\
\text { eassociada } \\
\text { eletroterapia nar } \\
\text { reabilitação do } \\
\text { paciente } \\
\text { hemiplegico. }\end{array}$ & 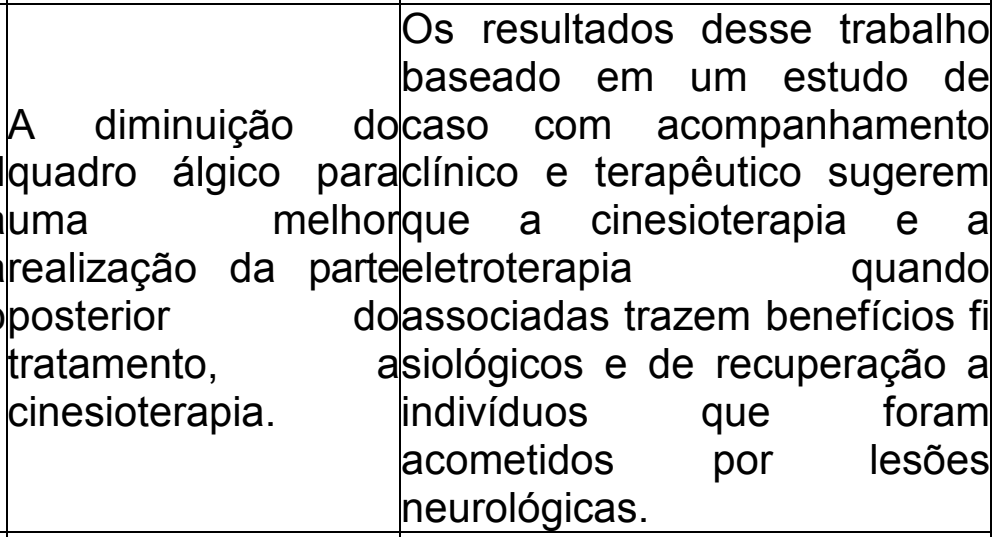 \\
\hline $\begin{array}{l}\text { Medeiros } \\
\text { al., (2014) }\end{array}$ & et. $\begin{array}{ll}\text { Efeito } & \text { da } \\
\text { terapia } & \text { de } \\
\text { espelho } & \text { por } \\
\text { meio } & \text { de } \\
\text { atividades } & \\
\text { padrões } \\
\text { motores } \\
\text { função } \\
\text { membro } \\
\text { superior } \\
\text { acidente }\end{array}$ & 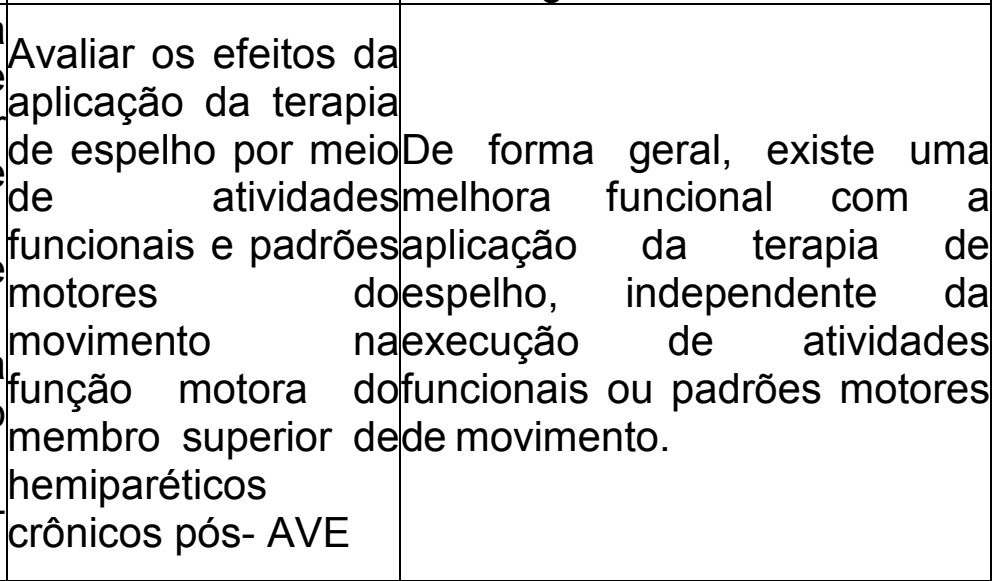 \\
\hline
\end{tabular}




\begin{tabular}{|c|c|c|}
\hline & $\begin{array}{l}\text { vascular } \\
\text { encefálico }\end{array}$ & \\
\hline $\begin{array}{l}\text { Leite et. al., } \\
(2009)\end{array}$ & 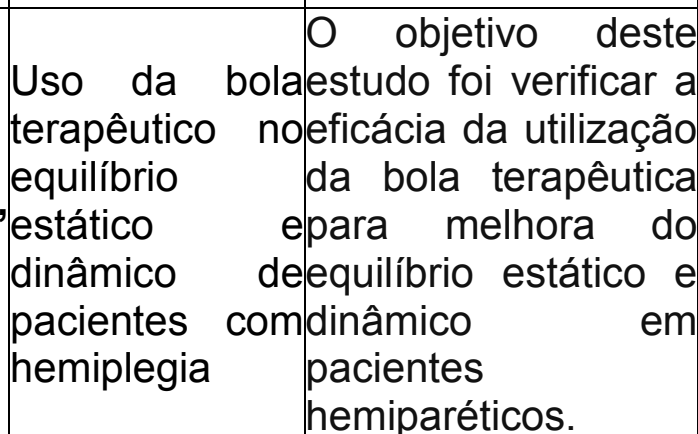 & 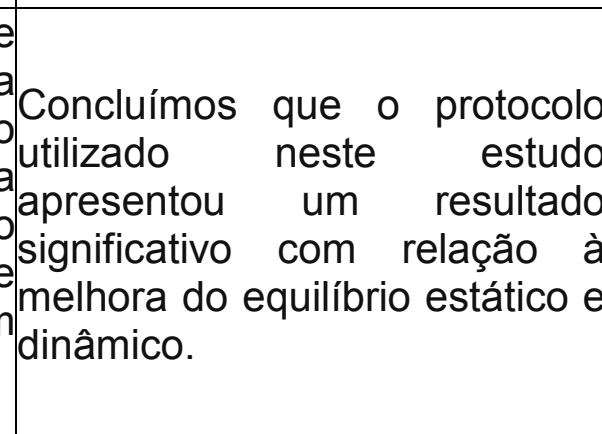 \\
\hline
\end{tabular}

Fonte: Dados da pesquisa (2019).

Com referência aos tipos de tratamentos, o quadro 2 exibe de forma precisa as quantidades dos diversos tratamentos utilizados nos artigos vistos.

Quadro 2 - Tipos de tratamentos utilizados nos artigos.

\begin{tabular}{|c|c|c|}
\hline TRATAMENTO & AUTOR/ANO & QUANTIDADE \\
\hline KINESIO TAPING & \begin{tabular}{|c|} 
Santos et. al., (2010) \\
Apolinário et. al., (2014)
\end{tabular} & 2 \\
\hline CRIOTERAPIA + CINESIOTERAPIA & Correia et. al., (2010) & 1 \\
\hline $\begin{array}{l}\text { CINESIOTERAPIA + } \\
\text { ELETROESTIMULACÃO }\end{array}$ & $\begin{array}{c}\text { Santos e Ferreira } \\
(2015)\end{array}$ & 1 \\
\hline TERAPIA NO ESPELHO & Medeiros et. al., (2014) & 1 \\
\hline BOLA TERAPÊUTICA & Leite et. al., (2009) & 1 \\
\hline ELETROTERAPIA + CRIOTERAPIA & $\begin{array}{l}\text { Felice; Ishizuka; } \\
\text { Amarilha (2011) }\end{array}$ & 1 \\
\hline
\end{tabular}

Fonte: Dados da pesquisa (2019).

\section{RESULTADOS E DISCUSSÕES}

Nesta pesquisa de Santos et al. (2010) foi utilizada na sua intervenção a Kinesio Taping, feito por kenso kase em 1996. A técnica é feita com uma bandagem elástica colocada em cima da pele, estendendo até $120-140 \%$ do seu tamanho regular, o que resulta em um mecanismo de pressão e força. É feita com um material resistente ao ar e a água, sendo capaz de ser utilizada por muitos dias. Assim, com 
este estudo, foi possível notar que todos os pacientes que possuíam subluxação no ombro após a aplicação do kinesio Taping mostraram uma redução da subluxação.

A pessoa acometida por esta patologia tem o sistema nervoso lesionado e um ou pelo menos todas as partes neurológicas que mantem a posição certa, e o úmero sua função também pode ser interrompida, havendo uma subluxação de ombro. Em vista disso, requer a utilização de tratamentos fisioterapêuticos, apresentado acima, melhorando a mobilidade do úmero.

No trabalho de Apolinário et al. (2014), fizeram avaliações contínuas no qual notou-se muitos fatores como a espasticidade, a força muscular e outros. Foi observado que existiu redução da espasticidade, aumentar da amplitude de movimento (ADM), da forca muscular no G1 que usou a cinesiologia quanto a kinesio tanping, enquanto que no G2 utilizou somente cinesioterapia, os números alcançados da espasticidade, ADM e força muscular mantiveram-se o mesmo durante todo o período de intervenção. Os resultados obtidos mostram que os dois tratamentos possuíram mudanças consideráveis e, são eficientes para este tipo de caso estudado. O estudo de Felice, Ishizuka e Amarilha (2011), foi usado a eletroestimulação e a crioterápica. Depois da avalição funcional com teste Timed Up and Go, no qual foi analisado o tempo que cada paciente levou para fazer um certo percurso, tiveram resultados bons para dois pacientes, sendo $84 \%$ no paciente 1 , de $86,3 \%$ no paciente 2 , mas, o paciente 3 não obteve variação. A avaliação eletromiografia foi capaz de reparar uma diminuição da atividade elétrica de quadríceps para os 3 pacientes.

Diante disso, reparou-se que os resultados da crioterápica destacou-se a estimulação elétrica. Mas, a crioterápica por ser um tratamento acessível, pode-se ter sido excelente, o paciente pode ter usufruído em casa no decorrer dos dias de intervalo entre a terapia.

Crioterapia é um tratamento que utiliza gelo para resfriar os tecidos e proporcionar uma diminuição da dor, dos espasmos musculares, do edema ou da inflamação. Estimação elétrica é usada no objetivo de reeducar o controle motor utilizado uma corrente elétrica posta na musculatura espástica. Essa técnica é utilizada pois recupera as atrofias ou desequilíbrios musculares para pacientes com 
imobilização e limitação de atividades, tendo também efeito analgésico. (FELICE; ISHIZUKA; AMARILHA, 2011).

Um estudo que utilizou também a crioterapia junto com a cinesioterapia foi de Correia et al. (2010). Este estudo teve resultados satisfatórios aos pacientes que tinham espasticidade, visto que as medidas realizadas antes e depois da intervenção com avaliação Goniométrica proporcionaram analisar uma diminuição do padrão postural flexor das articulações do punho metacarpofalangeas e interfalangeas, além do mais, notou-se uma melhoria na amplitude na extensão passiva dessas articulações. Nos estudos dos sete pacientes apenas um não teve progresso, mostrando espasticidade, vale lembrar que antes do tratamento era quatro pacientes que tinham espasticidade.

A crioterapia aplicada corretamente e de forma contínua diminui a espasticidade pelo fato e minimizar a neurotransmissão de impulsos aferente e eferentes, além de que, observa-se que a crioterapia deve ser usada em conjunto com diversas terapia para ter um resultado bom.

De acordo com Leite et al. (2009), utiliza-se o tratamento com a bola suíça na sua pesquisa, está se mostra bastante adequado para tratar hemiplegia e hemiparesia, na primeira é a paralisia de metade sagital (esquerda ou direita) do corpo e a segunda é uma sequela que dá- se com a alteração da marcha, dificultando o paciente de fazer suas atividades funcionais como de deambular e de auto cuidar.

Tendo vários tipos de tratamento dentro da fisioterapia que podem ser utilizados para tratar do AVC, diversos tipos, obtendo resultados positivos. A fisioterapia que pode ajudar de forma eficaz no tratamento desses pacientes abandonados em camas e cadeira de rodas sem ter uma chance de reabilitação e sem facilitar sua inclusão na sociedade.

A cinesiologia tem como objetivo fazer com que os movimentos que eram comuns na vida do paciente sejam reaprendidos e o melhorar a capacidade motora e com maior rapidez. Com relação aos exercícios usados Santos e Ferreira (2015) dizem que nas sessões o indivíduo foi ensinado para fazer a protrusão, abdução, flexão, adução e extensão de ombro de modo ativo e assistida, além, mobilização 
passiva em vários grupos musculares nos MMII e MMSS e alongamento na musculatura encurtada.

Conforme Medeiros et al. (2014) é usada a terapia espelho (TE) que são exercícios para o membro acometido superior usando um espelho entre os dois membros com o paciente de modo sagital. Está sendo muito utilizada em tratamento pós AVC e tem um baixo custo com uma fácil utilização.

O objetivo é reeducar o cérebro realizando movimentos com o membro saudável, que é refletido no espelho, assim, são interpretados de que é o membro comprometido que está se movimentando. Isso proporciona ao individo uma redescoberta e favorece no ganho de mobilidade e de coordenação do membro.

\section{CONSIDERAÇÕES FINAIS}

Esta pesquisa apresentou os resultados das técnicas mais eficazes na reabilitação de pacientes pós-AVC, para melhorar a funcionalidade destes pacientes. Esses estudos foram comprovados pelo o meio de analise através de escalas de avaliações, testes específicos e por pesquisa em forma de questionário. Tendo a necessidade de ampliar os estudos que acompanhem os tratamentos e as terapias desses indivíduos com amostras de maior para, desta maneira, conseguir resultados mais corretos.

O kinesio Taping e eletroestimulação foram os tratamentos mais utilizadas dentro dos artigos, mesmo esses sendo os mais utilizados não podemos esquecer dos benefícios dos demais. Todos os estudos citados alcançaram resultados satisfatórios, além do mais, trouseram o conhecimento da variedade de tratamentos fisioterapêuticos, que podem melhorar as sequelas de pacientes pós-AVC, também podendo ser utilizadas de forma conjunta, para potencializar os resultados, sendo gratificante para os pacientes perceber que houve uma redução dos seus sintomas rapidamente.

Para diferenciar suas principais dificuldades e limitações é de grande relevância que os fisioterapeutas envolvidos no procedimento de reabilitação 
procurem ouvir mais os pacientes e os cuidadores, para escolher o melhor tratamento tornando-o menos desagradável.

\section{REFERÊNCIAS BIBLIOGRÁFICAS}

ABRAMCZUK, B.; VILLELA, E. A luta contra o AVC no Brasil. Rev. ComCiência, n.109, ISSN 1519-7654. 2009.

APOLINARIO, E. S.; SATOS , E. F. S.;CARNEIRO,J. N. P; FERNNDES, C. D. M.; SOUZA, R. A. S. Kinesio Taping como auxiliar na recuperação de pacientes pós- AVC no membro superior parético: Um ensaio clinico. Revista interfaces: Saúde, Humanas e Tecnológica, Juazeiro do Norte - CE, v. 2, n. 6, 2014.

CORREIA, A.C.S.;SILVA, J.D.S.; SILVA, L.V.C.;OLIVEIRA, D.A.; CABRAL, E.D. Crioterapia e cinesioterapia no membro superior espástico no acidente vascular cerebral. Fisioter. Mov.,Curitiba, v. 23, n. 4, p. 555-563, 2010.

BOCCHI, S. C. M. Vivenciando a sobrecarga ao vir-a-ser um cuidador familiar de pessoa com acidente vascular cerebral (AVC): análise do conhecimento. Rev. Latino-Am. Enfermagem, Ribeirão Preto, v. 12, n. 1, p. 115-121, Fev. 2004. Disponível em: $<$ http://www.scielo.br/scielo.php?script=sci_arttext\&pid=S0104-

$11692004000100016 \&$ Ing=en\&nrm=iso>. Acesso em: 18 out. 2018.

BOTELHO, T. de S. et al. Epidemiologia do Acidente Vascular no Brasil. v. 16, n.2, p. 117,2016 .

FERNANDES, S.G.G.; LOPES, J.M.; CACHO, E.W.A.; CACHO, R.O. Efeito da terapia de espelho por meio de atividades funcionais e padrões motores na função do membro superior pós-acidente vascular encefálico. Fisioterapia Pesquisa, v.21, n.3, p.264-270, 2014.

FELICE, T.D.; ISHIZUKA, R.O.R,: AMARILHA, J.D. Eletroestimulação e crioterapia para espasticidade em pacientes acometidos por acidente vascular cerebral. Rev Neurociencias; 19:77-84. MEDEIROS, C.S.P.; 2011.

GARRITANO, C.R.; LUZ, P.M.; PIRES, M.L.E.; BARBOSA, M.T.S.; BATISTA, K.M. Análise da tendência da mortalidade por acidente vascular cerebral no Brasil no século XXI. Arq. Bras. Cardiol, 98(6):519-527. 2012.

LEITE, N.N. et al. Uso da bola terapêutica no equilíbrio estático e dinâmico de pacientes com hemiparesia. Fisioterapia em Movimento, Curitiba, v. 22, n. 1, p. 121-31, 2009.

LEVY, J.A.; OLIVEIRA, A. S. B. Reabilitação em doença neurológica: Guia terapêutica prático. São Paulo, 2003.

MENDES, K. dal S. et al. Revisão integrativa: método de pesquisa para a incorporação de evidências na saúde e na enfermagem. Texto \& Contexto Enfermagem, v. 17, n. 4, p. 758764, 2008.

ROLIM, C. L. R. C.; MARTINS, M. Qualidade do cuidado ao acidente vascular cerebral isquêmico no SUS. Cad. Saúde Pública [online]. 2011, vol.27, n.11, p.2106-2116. Disponível em:<http://dx.doi.org/10.1590/S0102-311X2011001100004>. Acesso em: 28 out. 2018. 
SANTOS, J.C.C. et al. A influência da Kinesio Taping no tratamento da subluxação de ombro no Acidente Vascular Cerebral. Rev Neurocienc; 18(3):335-40. 2010.

SANTOS, O. L. C. L. A.; FERREIRA, A. S. Cinesioterapia neuro- funcional associada a eletroterapia na reabilitação do paciente hemiplégico. Fisioterapia ser, Rio de janeiro, v.10, n. 2, 2015. 